\title{
Artificial Photosynthesis Would Unify the Electricity-Carbohydrate- Hydrogen Cycle for Sustainability
}

\author{
Yi-Heng Percival Zhang, ${ }^{1,2,3}$
}

\author{
${ }^{1}$ Biological Systems Engineering Department, Virginia Polytechnic Institute and State \\ University (Virginia Tech), 210-A Seitz Hall, Blacksburg, Virginia 24061, USA \\ ${ }^{2}$ Institute for Critical Technology and Applied Science (ICTAS), Virginia Polytechnic Institute \\ and State University, Blacksburg, Virginia 24061, USA \\ ${ }^{3}$ DOE BioEnergy Science Center (BESC), Oak Ridge, Tennessee 37831, USA
}

Email: ypzhang@vt.edu

Tel: (540) 231-7414 [O], Fax: (540) 231-3199 


\begin{abstract}
Sustainable development requires balanced integration of four basic human needs - air $\left(\mathrm{O}_{2} / \mathrm{CO}_{2}\right)$, water, food, and energy. To solve key challenges, such as $\mathrm{CO}_{2}$ fixation, electricity storage, food production, transportation fuel production, water conservation or maintaining an ecosystem for space travel, we wish to suggest the electricity-carbohydrate-hydrogen (ECHo) cycle, where electricity is a universal energy carrier, hydrogen is a clean electricity carrier, and carbohydrate is a high-energy density hydrogen $\left(14.8 \mathrm{H}_{2}\right.$ mass\% or 11-14 MJ electricity output/kg)carrier plus a food and feed source. Each element of this cycle can be converted to the other reversibly \& efficiently depending on resource availability, needs, and costs. In order to implement such cycle, here we propose to fix carbon dioxide by electricity or hydrogen to carbohydrate (starch) plus ethanol by cell-free synthetic biology approaches. According to knowledge in the literature, the proposed artificial photosynthesis must be operative. Therefore, collaborations are urgently needed to solve several technological bottlenecks before large-scale implementation.
\end{abstract}

Keywords: artificial photosynthesis, biofuels, cell-free synthetic biology, climate change, $\mathrm{CO}_{2}$ fixation, electricity storage, food production, hydrogen, ecosystem for space travel, water conservation 
Sustainable development is a pattern of resource use that aims to meet human needs while preserving the environment. As the human population grows and its needs and desires (e.g., food, energy) expand, the problem of sustaining civilization or even improving the quality of life is challenging for scientists and engineers.

To address sustainability challenges associated with air, water, food, and energy, I propose to construct the electricity, carbohydrate, and hydrogen (ECHo) cycle (Fig. 1). In this cycle, electricity is difficult to store but it is a universal energy carrier that can be produced from nonrenewable primary energy sources (e.g., fossil fuels or nuclear energy) or renewable primary energy sources (e.g., solar, wind, biomass, etc.); hydrogen, a clean electricity carrier that can be converted to electricity by fuel cells with high efficiency and without pollutants, is difficult and costly to store and distribute; solid carbohydrate, which is a high-density hydrogen carrier and food source, can be easily produced, stored, and distributed.

To implement the ECHo cycle, we must efficiently fix $\mathrm{CO}_{2}$ in the form of carbohydrates with an energy input of electricity or hydrogen (Fig. 1). When these technologies are developed, we can address the below a number of challenges associated with sustainability: (i) fixing $\mathrm{CO}_{2}$ to mitigate climate change, (ii) producing food from $\mathrm{CO}_{2}$ and water through artificial photosynthesis, (iii) generating sustainable transportation biofuel from $\mathrm{CO}_{2}$ and electricity, (iv) storing electricity as a form of chemical compound(s) on a large scale, (v) conserving water as compared to natural plant photosynthesis, (vi) storing hydrogen in the form of carbohydrate and biofuels, and (vii) constructing a sustainable ecosystem for space travel. 
We have demonstrated the feasibility of high-yield generation of hydrogen from carbohydrates and water mediated by synthetic pathway biotransformations (SyPaB) as $\mathrm{C}_{6} \mathrm{H}_{10} \mathrm{O}_{5}(\mathrm{aq})+7 \mathrm{H}_{2} \mathrm{O}$ (l) $\rightarrow 12 \mathrm{H}_{2}$ (g) $+6 \mathrm{CO}_{2}$ (g) [1, 2]. Therefore, we have proposed to use carbohydrates as a highdensity hydrogen carrier to solve the challenges associated with hydrogen production, storage, and distribution, as well as safety concerns [3]. A future high-efficiency transportation system would be based on a hybrid of an on-board or off-board sugar-to-hydrogen bioreformer, hydrogen-to-electricity proton electron membrane fuel cells (PEMFCs), and an electric motor [4]. If the obstacles to the sugar-to-hydrogen technology are addressed as expected [3, 5], this technology would answer a key question - What will replace cheap oil -- and when? [6]. Also, electricity can be generated from carbohydrate directly through biofuel cells [7-9].

In this article, I propose two novel artificial photosynthesis pathways, based on engineering design principles and available knowledge, for closing the ECHo cycle.

\section{Concept: Artificial Photosynthesis Mediated by Cell-free SyPaB}

In nature, carbon dioxide can be biologically fixed by plants, microorganisms, and animals. Most plants fix carbon dioxide by using chloroplasts through the reductive pentose-phosphate cycle, i.e., the Calvin-Benson cycle $[10,11]$. Some plants adapted to arid conditions utilize Crassulacean acid metabolism (CAM photosynthesis) [12]. Microorganisms can fix $\mathrm{CO}_{2}$ through the reductive citric acid cycle [13], the reductive acetyl-CoA pathway (Wood-Ljungdahl pathway) [14, 15], or the 3-hydroxypropionate pathway [16]. Sometimes, animal tissues (such as liver cells) have been found to fix $\mathrm{CO}_{2}$ in order to produce cell constituents (e.g., glycogen) $[17,18]$. 
Different from the natural $\mathrm{CO}_{2}$ fixation processes, I design a novel cell-free $\mathrm{CO}_{2}$ fixation process by utilizing a number of purified enzymes and co-enzymes:

$$
8 \mathrm{CO}_{2}(\mathrm{~g})+8 \mathrm{H}_{2} \mathrm{O}(\mathrm{l})+\text { electricity } \rightarrow \mathrm{C}_{6} \mathrm{H}_{10} \mathrm{O}_{5}(\mathrm{~s})+\mathrm{C}_{2} \mathrm{H}_{6} \mathrm{O}(\mathrm{l})+9 \mathrm{O}_{2}(\mathrm{~g})
$$

where the inputs are $\mathrm{CO}_{2}$, water, and electricity; the outputs are amylose (linear starch), ethanol, and oxygen.

Figure 2 presents the 21-enzyme synthetic enzymatic pathway, including six modules (see SOM Table 1 for the step-by-step reaction),

(1) Electrochemical NADH regeneration (Eq. 2) [19-21],

$$
18 \mathrm{NAD}^{+}+18 \mathrm{H}_{2} \mathrm{O}+\text { electricity } \rightarrow 18 \mathrm{NADH}+18 \mathrm{H}^{+}+9 \mathrm{O}_{2}
$$

(2) $\mathrm{CO}_{2}$ fixation to formaldehyde $\left(\mathrm{CH}_{2} \mathrm{O}\right)$ mediated by formate dehydrogenase and formaldehyde dehydrogenase (Eq. 3) [22-24],

$$
9 \mathrm{CO}_{2}+18 \mathrm{NADH}+18 \mathrm{H}^{+} \rightarrow 9 \mathrm{CH}_{2} \mathrm{O}+9 \mathrm{H}_{2} \mathrm{O}+18 \mathrm{NAD}^{+}
$$

(3) conversion of formaldehyde to fructose-6-phosphate (f6p) by 3-hexulose-6-phosphate synthase and hexulose phosphate isomerase from the ribulose monophosphate pathway (Eq. 4) $[25,26]$,

$$
9 \mathrm{CH}_{2} \mathrm{O}+9 \mathrm{ru} 5 \mathrm{p} \rightarrow 9 \mathrm{f} 6 \mathrm{p}
$$

(4) ribulose-5-phoshate (ru5p) regeneration by the eight enzymes from the non-oxidative pentose phosphate pathway (Eq. 5) [2, 10, 11],

$$
8 \mathrm{f} 6 \mathrm{p}+2 \mathrm{ATP}+\mathrm{P}_{\mathrm{i}} \rightarrow 9 \text { ru5p }+\mathrm{g} 3 \mathrm{p}+2 \mathrm{ADP}+\mathrm{P}_{\mathrm{i}}
$$

(5) ethanol production from glyceraldehydes-3-phosphate (g3p) by the seven enzymes from the glycolysis and ethanogenesis pathway (Eq. 7) [10, 11], 


$$
\mathrm{g} 3 \mathrm{p}+\mathrm{P}_{\mathrm{i}}+2 \mathrm{ADP} \rightarrow \mathrm{C}_{2} \mathrm{H}_{6} \mathrm{O}+\mathrm{CO}_{2}+2 \mathrm{ATP}
$$

(6) starch (amylose, a linear $\alpha$-1,4-glucosidic bond starch) lengthening reaction mediated by starch phopshophorylase along with phosphoglucose isomerase and phosphoglucomutase (Eq.

6) $[2,10,11]$,

$$
\mathrm{f} 6 \mathrm{p}+\left(\mathrm{C}_{6} \mathrm{H}_{10} \mathrm{O}_{5}\right)_{\mathrm{n}} \rightarrow\left(\mathrm{C}_{6} \mathrm{H}_{10} \mathrm{O}_{5}\right)_{\mathrm{n}+1}+\mathrm{P}_{\mathrm{i}}
$$

The combination of Equations 2-7 results in Eq. 1.

The overall reaction must be operative since (1) nearly each reaction is reversible, except 6phosphofructokinase and pyruvate kinase, which control the overall reaction direction (SOM Table 1) and (2) each of the combined equations (Eqs. 2-7) involving several enzymatic steps have been implemented successfully in the literature. This process can be driven forward the desired products since (i) high concentration $\mathrm{CO}_{2}$ from a power station can saturate the reaction solution, (ii) the amylose lengthening reaction occurs on non-reducing ends of the solid product (amylose) and amylose is more insoluble in the presence of ethanol, and (iii) ethanol can be stripped from the aqueous phase. Since this designed pathway has balanced NAD(H) and ATP without any costly input, it would be operative and economically feasible in the future.

Similar to the above pathway design, hydrogen can replace electricity for $\mathrm{NAD}(\mathrm{P}) \mathrm{H}$ regeneration by using hydrogenase possibly coupled with NAD transhydrogenase (Eq. 8) [1, 2, 27, 28]

$$
18 \mathrm{NAD}(\mathrm{P})^{+}+18 \mathrm{H}_{2} \rightarrow 18 \mathrm{NAD}(\mathrm{P}) \mathrm{H}+18 \mathrm{H}^{+}
$$


The combination of Equations 3-7 and 8 leads to Equation 9.

$$
8 \mathrm{CO}_{2}(\mathrm{~g})+18 \mathrm{H}_{2}(\mathrm{~g}) \rightarrow \mathrm{C}_{6} \mathrm{H}_{10} \mathrm{O}_{5}(\mathrm{~s})+\mathrm{C}_{2} \mathrm{H}_{6} \mathrm{O}(\mathrm{l})+10 \mathrm{H}_{2} \mathrm{O}(\mathrm{l})
$$

The above pathway designs have some flexibility for different outputs, for example, production of lactic acid or other chemicals, depending on needs.

\section{Applications and Efficiencies}

The outputs of the ECHo cycle depend mainly on resource availability, needs, and conversion costs \& efficiencies. When electricity is cheap or is idle at midnight or needs to be stored, carbohydrate and biofuels can be generated by fixing high concentration carbon dioxide from power stations or $\mathrm{CO}_{2}$ storage sites (Fig. 3a). This artificial photosynthesis process has a theoretical energy efficiency of 106\% and an estimated practical efficiency of $80 \%$ (Table 1). It is worth noting that this photosynthesis process has a very high energy utilization efficiency although numerous other non-natural photosynthesis pathways may be designed according to the $\mathrm{SyPaB}$ design principles. The overall practical solar-to-carbohydrate efficiency is estimated to be $\sim 12 \%$ by combining solar cells ( $\sim 15 \%)$ and artificial photosynthesis ( $\sim 80 \%)$, much higher than the efficiencies for natural plant photosynthesis (theoretical, C3 plants, 4.6\% [29]; theoretical, C4 plants (6\%) [29]; and practical, 0.2-1.0\% [30]). Consequently, this technology would drastically decrease land use for the production of food and animal feed. Meanwhile, this process would decrease water use by at least 500 -fold because most water for plant photosynthesis is used for transpiration rather than biomass synthesis [31]. 
When carbohydrate is cheap (mainly isolated from plants), one of its large applications is transportation [3, 4]. As we discussed before, carbohydrate is a good hydrogen carrier for the generation of electricity through PEM fuel cells with high power density (Fig. 3b). For portable electronics that require low power densities, electricity can be generated directly from carbohydrate through enzymatic biofuel cells [8, 9] (Fig. 3c).

Most times, hydrogen is more costly than electricity and carbohydrate [3]. It would be economically prohibitive to produce carbohydrate from hydrogen. But in some conditions (e.g., space travel with enough hydrogen source or electricity), it would be vital to fix carbon dioxide by electricity or hydrogen for the production of food, regeneration of oxygen, and storage of hydrogen or electricity (Fig. 3d).

\section{Closing remarks}

Proof-of-concept experiments are urgently needed to be conducted but the science behind these novel pathways is crystal clear. The obstacles to board applications of the above technologies are attributed to (1) lack of stable standardized enzymes as building blocks in the pathways, (2) costly labile coenzymes, and (3) cofactor electrochemical regeneration. Currently, several thermophilic enzymes with enough stability (i.e., high mass-based total turn-over numbers of $>$ 200,000) have been obtained in our laboratory [32, 33]. The discovery and utilization of more thermostable enzymes suitable for cell-free SyPaB would be a new industrial enzymes gold mine [34]. Costly NAD(P) cofactors are expected to be replaced by low-cost and stable biomimetic analogues [35, 36] plus engineered oxidoreductases that can work on the biomimics 
[37]. Also, several enzymes in the pathways may need further engineering for tolerating toxic metabolites, or increasing enzyme stability, or decreasing product inhibition, or other properties.

Acknowledgments: The author was partially supported by the AFOSR, DOE BioEnergy Science Center (BESC), USDA Bioprocessing and Biodesign Center, and DuPont Young Professor Award. YPZ thanked Dr. Weidong Huang and Noppadon Sathitsuksanoh in checking the calculation of energy conversion efficiencies.

Competing interests: YHPZ is the inventor of these novel artificial photosynthesis pathways, which are covered under provisional patent application. 


\section{References}

[1] X. Ye et al., ChemSusChem 2, 149 (2009).

[2] Y.-H. P. Zhang et al., PLoS One 2, e456 (2007).

[3] Y.-H. P. Zhang, Energy Environ. Sci. 2, 272 (2009).

[4] Y.-H. P. Zhang, and J. A. Rollin, Nature Precedings 2009, 3725.1 (2009).

[5] Y.-H. P. Zhang, Biotechnol. Bioeng., DOI: 10.1002/bit.22630 (2010).

[6] R. A. Kerr, and R. F. Service, Science 309, 101 (2005).

[7] S. K. Chaudhuri, and D. R. Lovley, Nat. Biotechnol. 21, 1229 (2003).

[8] M. J. Cooney et al., Energy Environ. Sci. 1, 320 (2008).

[9] M. Moehlenbrock, and S. Minteer, Chem. Soc. Rev. 37, 1188 (2008).

[10] J. M. Berg, J. L. Tymoczko, and L. Stryer, Biochemistry (fifth edition) (W. H. Freeman \& Co., New York, 2002).

[11] D. L. Nelson, and M. M. Cox, Lehninger Principles of Biochemistry (third edition) (Worth Publishers, New York, 2000).

[12] A. Herrera, Ann. Bot. 103, 645 (2009).

[13] J. Pereto et al., Int. Microbiol. 2, 3 (1999).

[14] V. Muller, Appl. Environ. Microbiol. 69, 6345 (2003).

[15] H. Wood, FASEB J. 5, 156 (1991).

[16] I. A. Berg et al., Science 318, 1782 (2007).

[17] E. A. Evans, Jr., Science 96, 25 (1942).

[18] H. A. Krebs, Mol. Cell. Biochem. 5, 79 (1974).

[19] R. DiCosimo, C.-H. Wong, and G. M. Whitesides, J. Org. Chem. 46, 4622 (1981).

[20] S. K. Yoon et al., J. Am. Chem. Soc. 127, 10466 (2005).

[21] K. Vuorilehto, S. Lutz, and C. Wandrey, Bioelectrochemistry 65, 1 (2004).

[22] Kuwabata S, R. Tsuda, and H. Yoneyama, J. Am. Chem. Soc. 116, 5437 (1994).

[23] R. Obert, and B. C. Dave, J. Am. Chem. Soc. 121, 12192 (1999). 
[24] B. El-Zahab, D. Donnelly, and P. Wang, Biotechnol. Bioeng. 99, 508 (2008).

[25] N. Kato, H. Yurimoto, and R. K. Thauer, Biosci. Biotechnol. Biochem. 70, 10 (2006).

[26] I. Orita et al., J. Bacteriol. 188, 4698 (2006).

[27] L. Greiner et al., Green Chem. 5, 697 (2003).

[28] A. L. De Lacey et al., Biotechnol. Bioeng. 68, 1 (2000).

[29] X.-G. Zhu, S. P. Long, and D. R. Ort, Curr. Opin. Biotechnol. 19, 153 (2008).

[30] Y.-H. P. Zhang, J. Ind. Microbiol. Biotechnol. 35, 367 (2008).

[31] M. Hightower, and S. A. Pierce, Nature 452, 285 (2008).

[32] Y. Wang, and Y.-H. P. Zhang, Microb. Cell Fact. 8, 30 (2009).

[33] Y. Wang, and Y.-H. P. Zhang, J. Appl. Microbiol. 108, 39 (2010).

[34] Y.-H. P. Zhang, Microbe 4, 560 (2009).

[35] R. J. Ansell, and C. R. Lowe, Appl. Microbiol. Biotechnol. 51, 703 (1999).

[36] H. C. Lo, and R. H. Fish, Angew. Chem. Int. Ed. 41, 478 (2002).

[37] J. D. Ryan, R. H. Fish, and D. S. Clark, ChemBioChem 9, 2579 (2008).

[38] J. A. Turner, Science 305, 972 (2004).

[39] F. Barbir, PEM Fuel Cells: Theory and Practice (Academic Press, 2005).

[40] L. Carreette, K. A. Friendrich, and U. Stimming, Fuel Cells 1, 5 (2001). 


\section{Figure Legends}

Figure 1. Scheme of the electricity-carbohydrate-hydrogen (ECHo) cycle with primary energy sources and biomass utilization. Dot lines presents the proposed artificial photosynthesis pathways.

Figure 2. The artificial photosynthesis pathway for $\mathrm{CO}_{2}$ fixation powered by electricity. Enzyme list: \#1 FDH, formate dehydrogenase (EC 1.2.1.2); \#2 FADH, formaldehyde dehydrogenase (EC 1.2.1.46); \#3 HPS, 3-hexulose-6-phosphate synthase (EC 4.1.2.43); \#4 HPI, hexulose phosphate isomerase (EC 5.3.1.27); \#5 FKP, 6-phosphofructokinase (EC 2.7.1.11); \#6 ALD, aldolase (EC 4.1.2.13); \#7 TIM, triose-phosphate isomerase (EC 5.3.1.1); \#8 TK, transketolase (EC 2.2.1.1); \#9 TAL, transaldolase (EC 2.2.1.2); \#10 R5PI, ribose 5-phosphate isomerase (EC 5.3.1.6); \#11 Ru5PE, ribulose5-phosphate 3-epimerase (EC 5.1.3.1); \#12 GAPDH, glyceraldehyde-3-phosphate dehydrogenase (EC 1.2.1.12); \#13 PGK, phosphoglycerate kinase (2.7.2.3); \#14 PGCM, phosphoglycerate mutase (EC 5.4.2.1); \#15 ENO, enolase (EC 4.2.1.11); \#16 PK, pyruvate kinase (EC 2.7.1.40); \#17 PDC, pyruvate decarboxylase (EC 4.1.1.1); \#18 ADH, alcohol dehydrogenase (EC 1.1.1.1); \#19 PGI; phosphoglucose isomerase (EC 5.3.1.9); \#20 PGM, phosphoglucomutase (EC 5.4.2.2), and \#21 $\alpha \mathrm{GP}$, starch phosphorylase (EC 2.4.1.1). Compound and metabolite list: Med $_{\mathrm{red}}$, reduced mediator; Med $_{\mathrm{ox}}$, oxidized mediator; HCOOH, formate; HCHO, formaldehyde; ru5p, ribulose-5-P; hu6p, 3-hexulose-6-P; f6p, fructose-6-phosphate; f16p, fructose-1,6-bisphosphate; g3p, glyceraldehydes-3-phosphate; g6p, glucose6-phosphate; $\mathrm{G}_{\mathrm{n}}$, linear starch (amylose); $\mathrm{P}_{\mathrm{i}}$, free inorganic phosphate, and $\mathrm{C}_{2} \mathrm{H}_{6} \mathrm{O}$, ethanol.

Figure 3. Different applications of the ECHo cycles. 
Table 1. Conversion efficiencies of each direction conversion in the ECHo cycle.

\begin{tabular}{|c|c|c|c|c|}
\hline Conversion & $\begin{array}{l}\text { Theoretic } \\
\text { efficiency } \\
\text { [a] }\end{array}$ & Ref. & $\begin{array}{l}\text { Practical } \\
\text { efficiency } \\
\text { [a] }\end{array}$ & Ref. \\
\hline Electrolysis (electricity $\rightarrow \mathrm{H}_{2}$ ) & $102 \%$ & calculated & $\sim 70 \%$ & [38] \\
\hline PEM fuel cell ( $\mathrm{H}_{2} \rightarrow$ electricity) & $83 \%$ & [39] & 50-60\% & [40] \\
\hline $\begin{array}{l}\text { Biofuel cells (carbohydrate } \rightarrow \\
\text { electricity) }\end{array}$ & $94 \%$ & calculated & $\sim 10-80 \%$ & [7] \\
\hline Electricity $\rightarrow$ carbohydrate $^{[b]}$ & $106 \%$ & this study & $\sim 80 \%$ & estimated \\
\hline Carbohydrate $\rightarrow \mathrm{H}_{2}$ & $122 \%$ & [1-3] & $\sim 120 \%$ & {$[1-3]$} \\
\hline $\mathrm{H}_{2} \rightarrow$ carbohydrate ${ }^{[c]}$ & $82 \%$ & this study & $\sim 75 \%$ & estimated \\
\hline
\end{tabular}

[a] based on high heating values (standard enthalpy).

[b] $8 \mathrm{CO}_{2}+8 \mathrm{H}_{2} \mathrm{O}+$ electricity $\rightarrow \mathrm{C}_{6} \mathrm{H}_{10} \mathrm{O}_{5}+\mathrm{C}_{2} \mathrm{H}_{6} \mathrm{O}+9 \mathrm{O}_{2}$

[c] $8 \mathrm{CO}_{2}+18 \mathrm{H}_{2} \rightarrow \mathrm{C}_{6} \mathrm{H}_{10} \mathrm{O}_{5}+\mathrm{C}_{2} \mathrm{H}_{6} \mathrm{O}+10 \mathrm{H}_{2} \mathrm{O}$ 


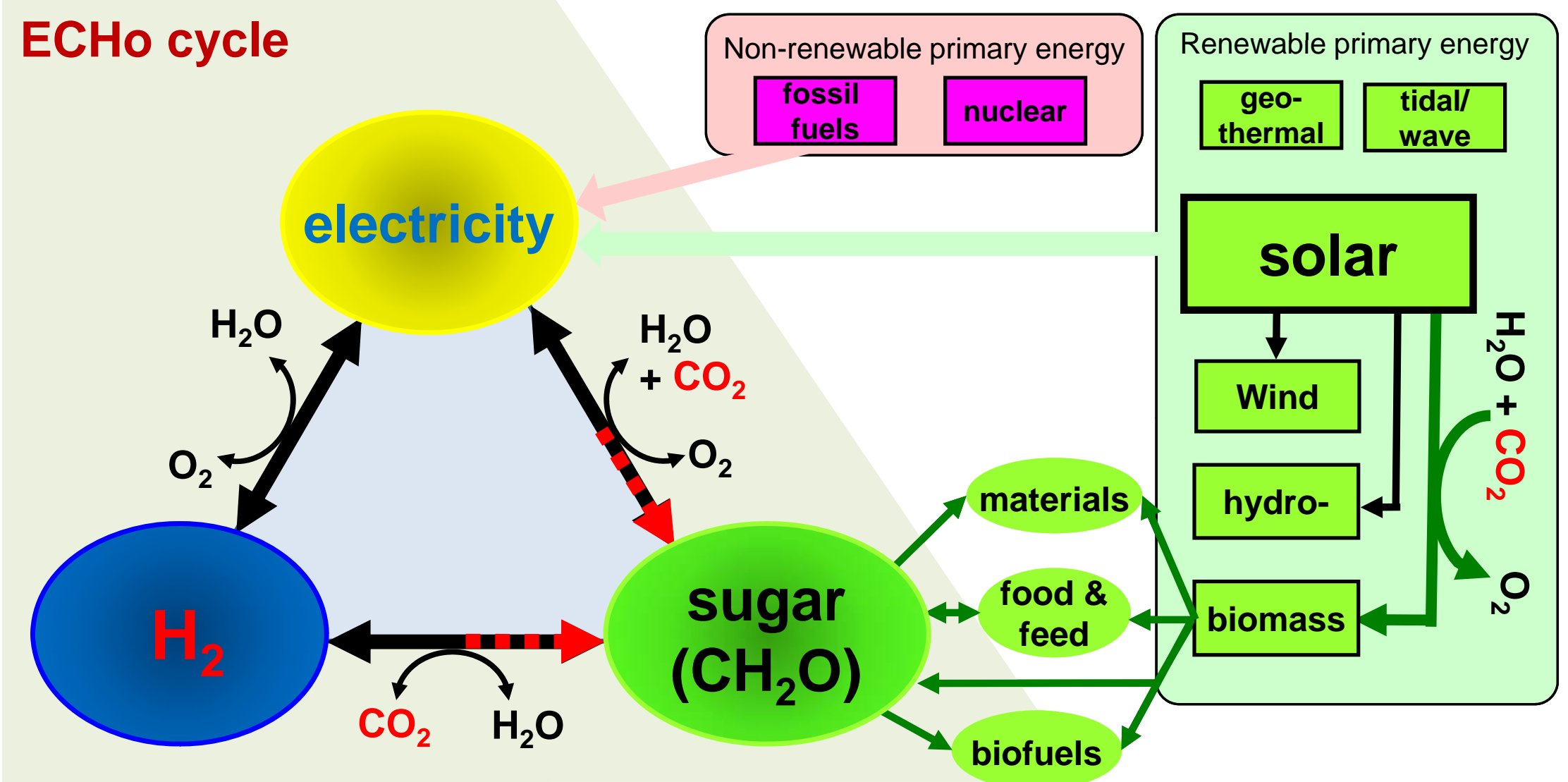

Fig. 1 


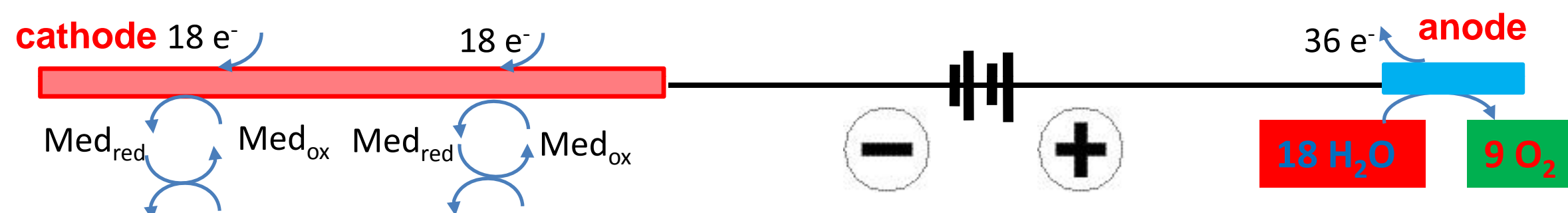

$9 \mathrm{NADH} 9 \mathrm{NAD}^{+} 9 \mathrm{NADH} 9 \mathrm{NAD}^{+}$
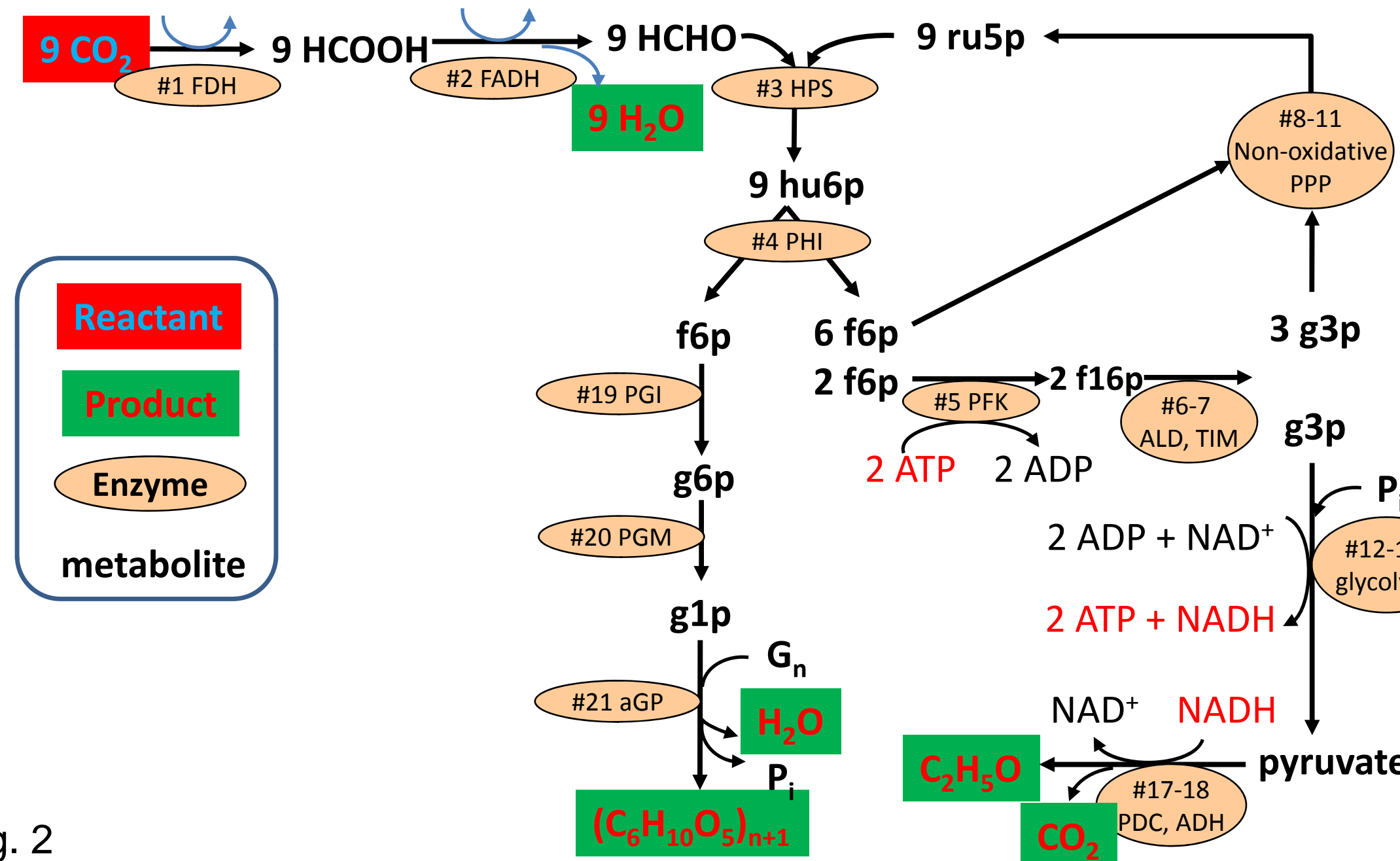

2 ATP 2 ADP ALD, TIM g3p

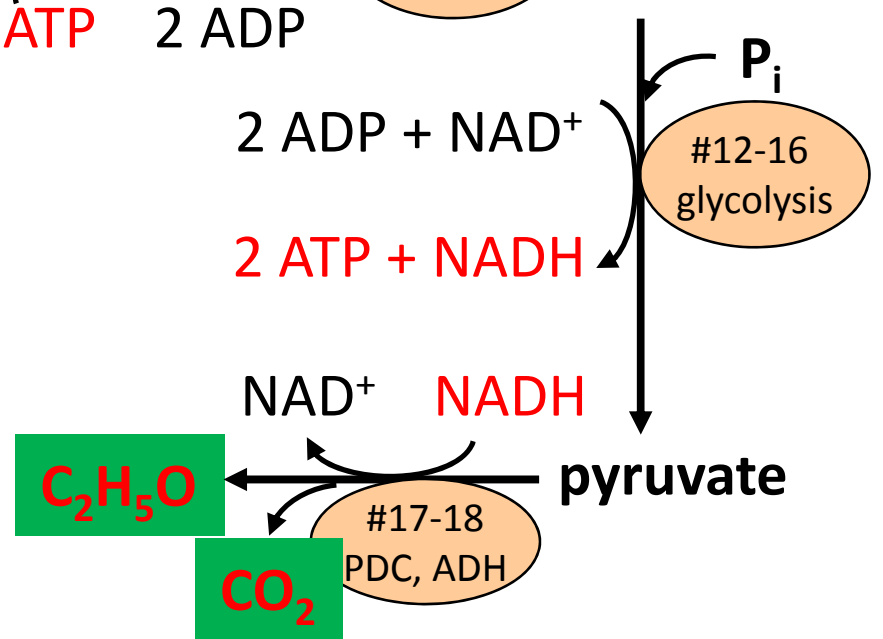

Fig. 2 
a
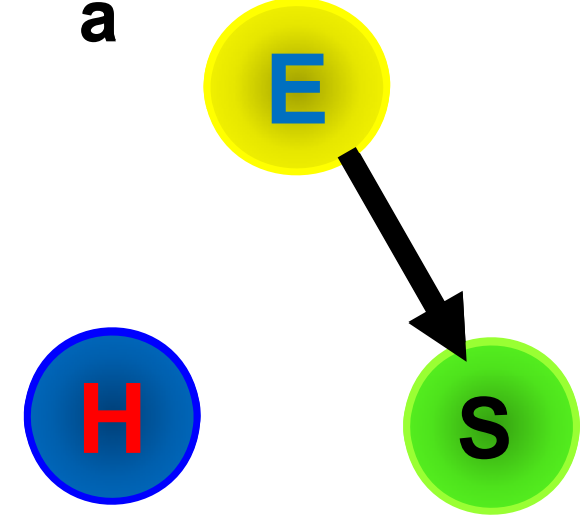

Electricity storage $\mathrm{CO}_{2}$ fixation Food production Water conservation

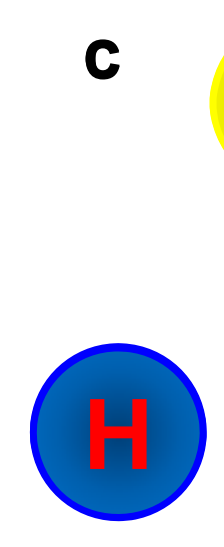

Portable electronics

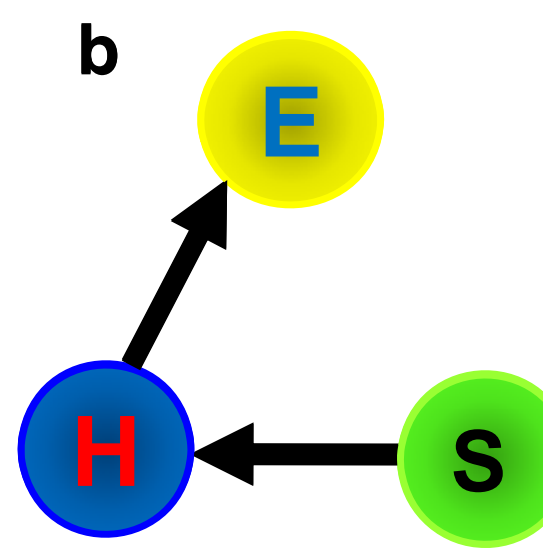

Transportation

Fig. 3

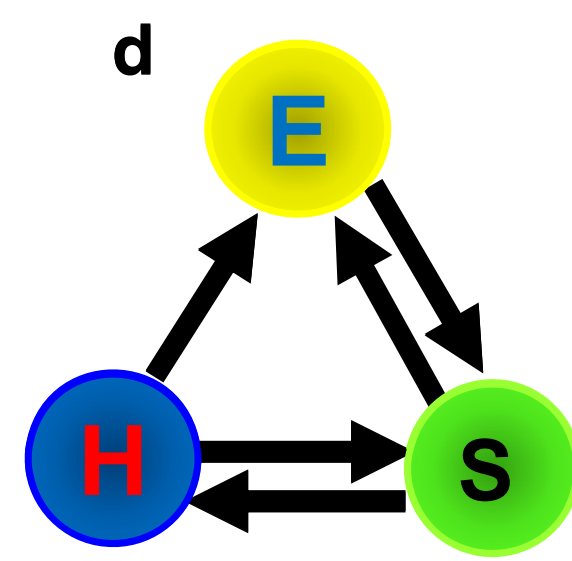

Space travel 


\title{
On-line Supplementary Materials
}

\begin{abstract}
Novel artificial photosynthesis would unify the electricity-carbohydratehydrogen (ECHo) cycle
\end{abstract}

\author{
Y.-H. Percival Zhang ${ }^{1,2,3}$ \\ ${ }^{1}$ Biological Systems Engineering Department, Virginia Polytechnic Institute and State \\ University (Virginia Tech), 210-A Seitz Hall, Blacksburg, Virginia 24061, USA \\ ${ }^{2}$ Institute for Critical Technology and Applied Science (ICTAS), Virginia Polytechnic Institute \\ and State University, Blacksburg, Virginia 24061, USA \\ ${ }^{3}$ DOE BioEnergy Science Center (BESC), Oak Ridge, Tennessee 37831, USA \\ Email: ypzhang@vt.edu
}

Tel: (540) 231-7414 [O], Fax: (540) 231-3199 
Figure S1. The synthetic artificial photosynthesis pathway for $\mathrm{CO}_{2}$ fixation powered by hydrogen.

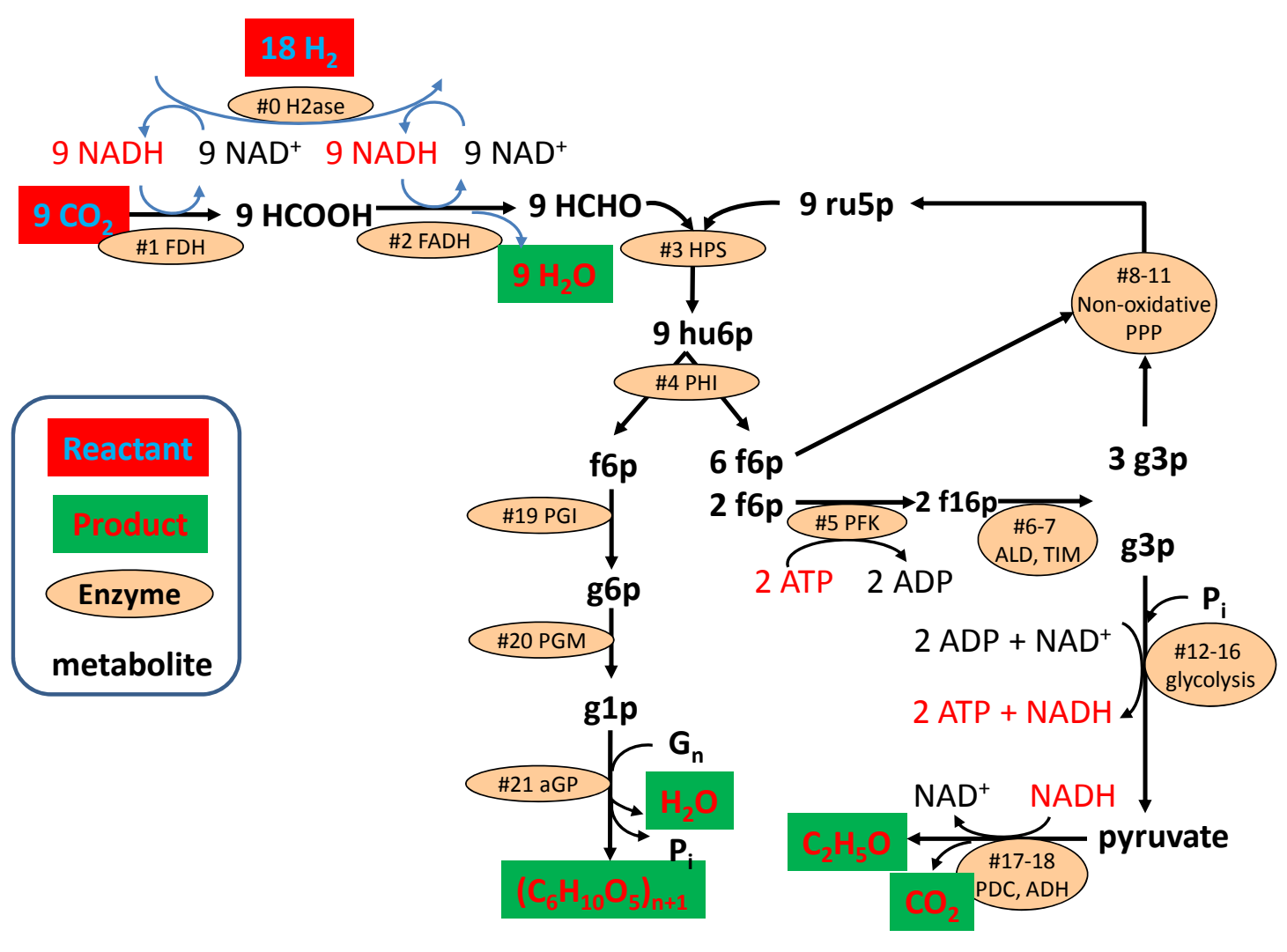


Table S1. The pathway for artificial photosynthesis powered by electricity $\left(8 \mathrm{CO}_{2}+8 \mathrm{H}_{2} \mathrm{O}+\right.$ electricity $\left.\rightarrow \mathrm{C}_{6} \mathrm{H}_{10} \mathrm{O}_{5}+\mathrm{C}_{2} \mathrm{H}_{6} \mathrm{O}+9 \mathrm{O}_{2}\right)$.

I. Electrochemical NADH regeneration: $\mathrm{NAD}^{+}+\mathrm{H}_{2} \mathrm{O}+$ electricity $\rightarrow \mathrm{NADH}+\mathrm{H}^{+}+1 / 2 \mathrm{O}_{2}$ Anode (oxidation)

Cathode (electron out)

$$
\begin{aligned}
\mathrm{NAD}^{+}+\mathrm{H}^{+}+2 \mathrm{e}^{-} \rightarrow \mathrm{NADH} \\
\mathrm{H}_{2} \mathrm{O} \rightarrow 1 / 2 \mathrm{O}_{2}+2 \mathrm{H}^{+}+2 \mathrm{e}^{-}
\end{aligned}
$$

\begin{tabular}{|c|c|c|c|c|c|}
\hline No. & E.C. & Enzyme Name & Reaction & pathway & Dir. \\
\hline \multicolumn{6}{|c|}{ II. $\mathrm{CO}_{2}$ fixation $9 \mathrm{CO}_{2}+18 \mathrm{NADH}+18 \mathrm{H}^{+} \rightarrow 9 \mathrm{CH}_{2} \mathrm{O}+9 \mathrm{H}_{2} \mathrm{O}+18 \mathrm{NAD}^{+}$} \\
\hline$\# 1$ & 1.2 .1 .2 & $\begin{array}{l}\text { formate dehydrogenase } \\
\text { (FDH) }\end{array}$ & $\mathrm{CO}_{2}+\mathrm{NADH}+\mathrm{H}^{+} \rightarrow$ formate $\left(\mathrm{CH}_{2} \mathrm{O}_{2}\right)+\mathrm{NAD}^{+}$ & methanogenesis & $\rightarrow \leftarrow$ \\
\hline$\# 2$ & 1.2 .1 .46 & $\begin{array}{l}\text { formaldehyde } \\
\text { dehydrogenase (FADH) }\end{array}$ & formate $+\mathrm{NADH}+\mathrm{H}^{+} \rightarrow$ formaldehyde $\left(\mathrm{CH}_{2} \mathrm{O}\right)+\mathrm{NAD}^{+}+\mathrm{H}_{2} \mathrm{O}$ & methanogenesis & $\rightarrow \leftarrow$ \\
\hline \multicolumn{6}{|c|}{ III. The ribulose monophosphate pathway $\left(9 \mathrm{CH}_{2} \mathrm{O}+9 \mathrm{ru} 5 \mathrm{p} \rightarrow 9 \mathrm{f6p}\right)$} \\
\hline \#3 & 4.1.2.43 & $\begin{array}{l}\text { 3-hexulose-6-phosphate } \\
\text { synthase (HPS) }\end{array}$ & ribulose-5-P (ru5p) + formaldehyde $\rightarrow$ 3-hexulose-6-P (hu6p) & $\begin{array}{l}\text { ribulose mono- } \\
\text { phosphate pathway }\end{array}$ & $\rightarrow \leftarrow$ \\
\hline$\# 4$ & 5.3.1.27 & $\begin{array}{l}\text { hexulose phosphate } \\
\text { isomerase (HPI) }\end{array}$ & 3-hexulose-6-P (hu6p) $\rightarrow$ fructose-6-phosphate (f6p) & $\begin{array}{l}\text { ribulose mono- } \\
\text { phosphate pathway }\end{array}$ & $\rightarrow \leftarrow$ \\
\hline \multicolumn{6}{|c|}{ IV. Branched pathways for the overall reaction $\left(8 \mathrm{f} 6 p+2 \mathrm{ATP}+\mathrm{P}_{\mathrm{i}} \rightarrow 9\right.$ ru5p $+\mathrm{g} 3 p+2$ ADP) } \\
\hline \multicolumn{6}{|c|}{$2 \mathrm{f} 6 \mathrm{p}+2$ ATP $\rightarrow 4 \mathrm{~g} 3 \mathrm{p}+2$ ADP } \\
\hline \#5 & 2.7.1.11 & $\begin{array}{l}\text { 6-phosphofructokinase } \\
\text { (PFK) }\end{array}$ & $\begin{array}{l}\text { fructose-6-phosphate (f6p) + ATP } \rightarrow \text { fructose-1,6-bisphosphate } \\
\text { (f16p) }\end{array}$ & glycolysis & $\rightarrow$ \\
\hline \#6 & 4.1 .2 .13 & $\begin{array}{l}\text { fructose-bisphophate } \\
\text { aldolase (ALD) }\end{array}$ & $\begin{array}{l}\text { fructose-1,6-bisphosphate (f16p) } \rightarrow \text { glyceraldehydes-3-phosphate } \\
\text { (g3p) + dihydroxacetone phosphate (dhap) }\end{array}$ & glycolysis & $\rightarrow \leftarrow$ \\
\hline \#7 & 5.3.1.1 & $\begin{array}{l}\text { triosephosphate } \\
\text { isomerase (TIM) }\end{array}$ & $\begin{array}{l}\text { dihydroxacetone phosphate (dhap) } \rightarrow \text { glyceraldehydes-3-phosphate } \\
\text { (g3p) }\end{array}$ & glycolysis & $\rightarrow \leftarrow$ \\
\hline \multicolumn{6}{|c|}{$6 \mathrm{f6p}+3$ g3p $\rightarrow 9$ ru5p } \\
\hline$\# 8$ & 2.2.1.1 & $\begin{array}{l}\text { transketolase } \\
\text { (TK) }\end{array}$ & $\begin{array}{l}\text { fructose-6-phosphate (f6p)+ glyceraldehyde-3-phosphate (g3p) } \rightarrow \\
\text { xylulose-5-phosphate (x5p) + erythrose-4-phosphate (e4p) } \\
\text { sedoheptulose-7-phosphate (s7p) + glyceraldehyde-3-phosphate } \\
(\mathrm{g} 3 p) \rightarrow \text { xylulose-5-phosphate (xu5p) + ribose-5-phosphate (r5p) }\end{array}$ & $\begin{array}{l}\text { pentose phosphate } \\
\text { pathway (PPP) }\end{array}$ & $\rightarrow \leftarrow$ \\
\hline$\# 9$ & 2.2.1.2 & $\begin{array}{l}\text { transaldolase } \\
\text { (TAL) }\end{array}$ & $\begin{array}{l}\text { fructose-6-phosphate (f6p)+ erythrose-4-phosphate (e4p) } \rightarrow \\
\text { sedoheptulose-7-phosphate (s7p) + glyceraldehyde-3-phosphate }\end{array}$ & PPP & $\rightarrow \leftarrow$ \\
\hline
\end{tabular}




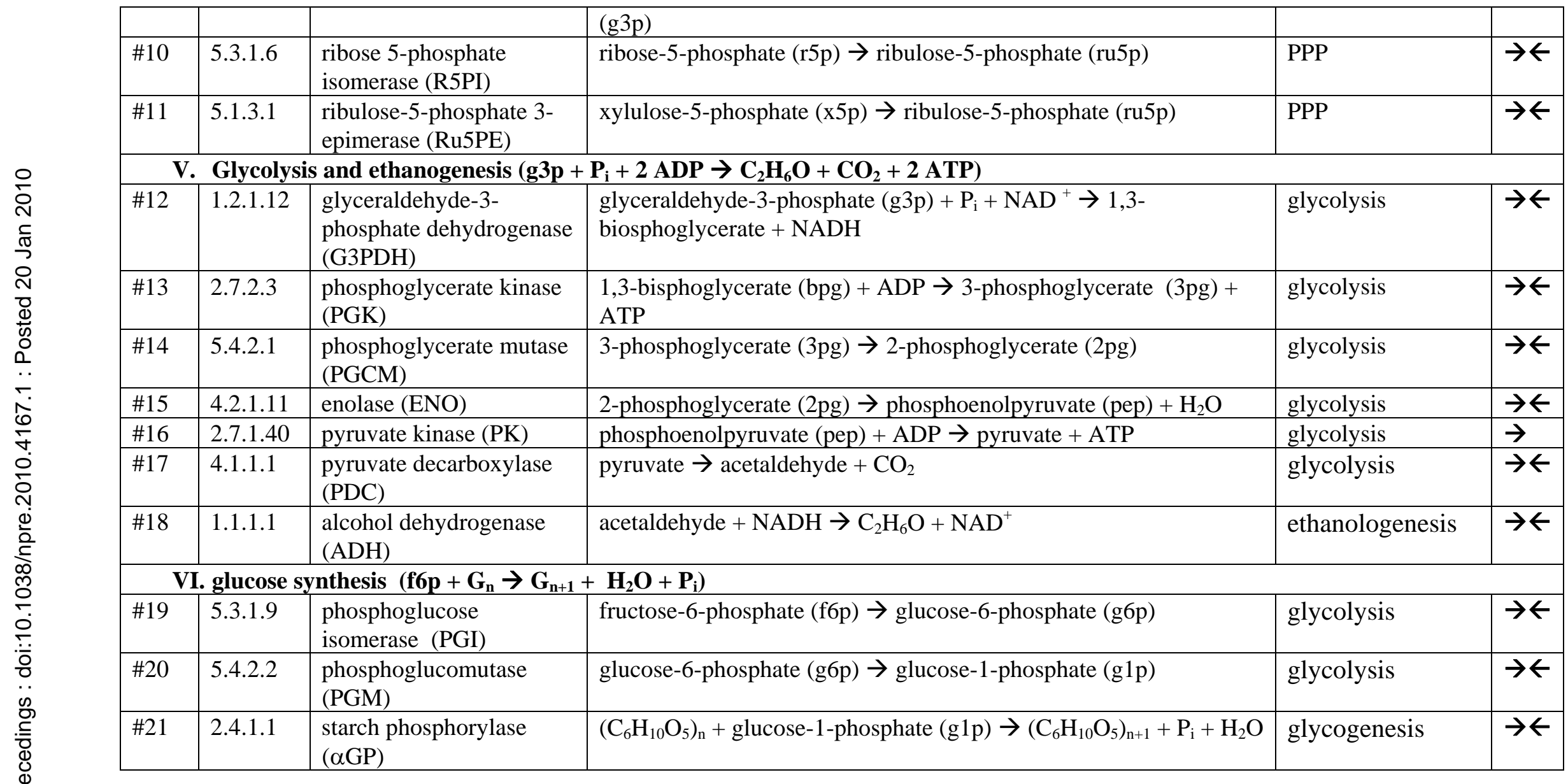


Table S2. The pathway of artificial photosynthesis powered from hydrogen $\left(8 \mathrm{CO}_{2}+18 \mathrm{H}_{2} \rightarrow\right.$ $\mathrm{C}_{6} \mathrm{H}_{10} \mathrm{O}_{5}+\mathrm{C}_{2} \mathrm{H}_{6} \mathrm{O}+10 \mathrm{H}_{2} \mathrm{O}$ ).

\begin{tabular}{|l|l|l|l|l|}
\hline No. & E.C. & Enzyme Name & Reaction & Dir. \\
\hline \multicolumn{2}{|c|}{ I. NADH regeneration } & $\mathrm{NAD}^{+}+\mathrm{H}_{2} \rightarrow \mathrm{NADH}+\mathrm{H}^{+}$ & $\leftarrow \rightarrow$ \\
\hline$\# 0$ & 1.12 .1 .3 & hydrogenase (H2ase) & \\
\hline
\end{tabular}

The remaining enzymes from \#1-21 are the same as the artificial photosynthesis pathway powered by electricity. 